\title{
Antiwear performance of polysiloxane-containing copolymers at oil/metal interface under extreme pressure
}

\author{
Sheng-Chung $\mathrm{Ni}^{\mathrm{a}}$, Ping-Lin Kuo ${ }^{\mathrm{a}}{ }^{*}$, Jen-Fin Lin ${ }^{\mathrm{b}}$ \\ a Department of Chemical Engineering, National Cheng Kung University, Tainan, Taiwan 70101, ROC \\ b Department of Mechanical Engineering, National Cheng Kung University, Tainan, Taiwan 70101, ROC \\ Received 5 February 2002; received in revised form 16 July 2002; accepted 16 July 2002
}

\begin{abstract}
Copolymers separately containing polysiloxane, thiophosphate-polysiloxane, thiophosphate-amino, and polysiloxane-amino groups were synthesized by free radical copolymerization. The antiwear behaviors of the synthesized copolymers as an oil additive in base oil were assessed with a wear tester by measuring the oil temperature, frictional coefficient, and electrical contact resistance between the two metal surfaces. Thiophosphate-containing copolymers were noted to limit the increase in the oil temperature and reduce the frictional coefficient by adsorbing the thiophosphate group onto the metal surfaces and then reacting on those surfaces. The oil temperature and the frictional coefficient of thiophosphate-polysiloxane-containing copolymer were lower than that of other synthesized copolymers before chemical adsorption by the thiophosphate group, which was resulted from the protection by the polysiloxane structure. The amino-polysiloxane-containing copolymer that settled on the metal surfaces by adsorbing the amino group reduced the frictional coefficient and limited the increase in the oil temperature caused by the presence of the polysiloxane group. Nevertheless, the amino-polysiloxane-containing copolymer physically adsorbed onto the metal surfaces at a low temperature and desorbed from the metal surfaces with increasing oil temperature.
\end{abstract}

(C) 2002 Elsevier Science B.V. All rights reserved.

Keywords: Antiwear additive; Polysiloxane-containing copolymer; Tribology

\section{Introduction}

Lubricants are used mainly to reduce a frictional coefficient, and its capacity is closely related to the effectiveness of antiwear (AW) additives and the properties of extreme pressure (EP) additives. The effectiveness of additives is a function of physical adsorption and subsequent chemical reaction on a metal surface [1,2]. This work investigates whether the action of AW additives, which contain phosphorus-sulfur compounds, is based on first absorbing polar substances onto metal surfaces, where the subsequent reaction with the metal in the friction zone under the effect of heat and mechanical energy creates a phosphorus-sulfur rich reaction layer [3-10].

Previous works examined the adsorption phenomena of amino-containing copolymers from the perspective of AW performance. The measurement results indicated that an amino group in copolymers could physically adsorb onto a metal surface [11]. Silicone moiety is frequently included in the chemical structures of AW additives because silicone compounds are stable at high temperatures [12]. Further-

\footnotetext{
* Corresponding author. Tel.: +886-6-2757575; fax: +886-6-2762331.
} E-mail address: pluko@mail.ncku.edu.tw (P.-L. Kuo). more, if an EP additive is a high molecular weight polymer, then the layer formed on the metal surface will be much thicker and more effective in preventing contact between two metal surfaces [13-16].

This investigation synthesized numerous types of thiophosphate, polysiloxane, amino, and carboxylate-containing copolymers using free radical polymerization. An AW machine was employed to examine the AW characteristics of the oil containing added synthesized copolymers by measuring the oil temperature, frictional coefficient, and electrical contact resistance between two metal surfaces with a $200 \mathrm{~kg}$ load and $700 \mathrm{rpm}$ rotary velocity over $2 \mathrm{~h}$ [17]. The measurement results demonstrate that the thiophosphate-containing copolymer can limit the rise in oil temperature and reduce the frictional coefficient between the two metal surfaces by the physical adsorption and chemical reaction of the thiophosphate group onto the two surfaces. Furthermore, the oil temperature and frictional coefficient of the metal surfaces, when thiophosphate-polysiloxane-containing copolymer was added to the oil, were both lower than for the other synthesized copolymers before chemical adsorption because the two surfaces were protected from wear by the presence of polysiloxane group. The amino-polysiloxane-containing copolymer physically adsorbed onto the metal surfaces at 
a low temperature by the amino group and desorbed from the metal surfaces as the oil temperature rose. Furthermore, the amino-polysiloxane-containing copolymer that settled on the metal surfaces reduced the frictional coefficient and limited the increase in the oil temperature caused by the presence of the polysiloxane group.

\section{Experimental}

\subsection{Materials}

2-Hydroxyethylmethacrylate (2-HEMA, Arcos), triethylamine (TCI), diethyl chlorothiophosphate (Aldrich), siloxane oligomer (SY-231, Wacker), tripropyl titanate (TPT, ICI), stearyl acrylate (SA) (Aldrich), 2.2' -azobisisobutyronitrile (AIBN, TCI) and 2-dimethylamino ethylacrylate (TCI) were used as purchased. All the solvents were reagent grade or were purified using standard methods. The base oil (grade $\mathrm{HN}$, viscosity index $=95$, viscosity at $40^{\circ} \mathrm{C}=119-$ 129 cst) was obtained from Chinese Petroleum Corporation. Finally, the frictional pairs used in the AW tests were constructed from FC40 cast iron.

\subsection{Synthesis of poly (SAM-DAEA-SA) (SiNS)}

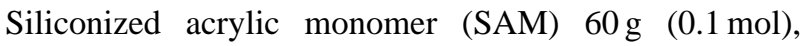
2-dimethylamino ethylacrylate (DAEA) $14.3 \mathrm{~g}$ (0.1 mol), and SA $97.2 \mathrm{~g}(0.3 \mathrm{~mol})$ were dissolved in $100 \mathrm{~g}$ toluene, in a $300 \mathrm{ml}$ four-necked reactor equipped with a mechanical stirrer. After the solution was heated to $85^{\circ} \mathrm{C}$, a solution of AIBN $(1.5 \mathrm{~g})$ and toluene $50 \mathrm{~g}$ was slowly transferred into the solution, the reaction was blanketed in a low-pressure nitrogen stream for $30 \mathrm{~min}$ and was then stirred at $95^{\circ} \mathrm{C}$ for $4 \mathrm{~h}$. The solvent was removed by rotary evaporation and the residue was poured in $300 \mathrm{~g}$ acetone. The precipitated copolymer was washed with acetone and dried at room temperature in a vacuum for $24 \mathrm{~h}$.

\subsection{Synthesis of poly \\ (DMP-2-dimethylaminoethylacrylate-SA) (PNS)}

Polymerization was conducted using a method similar to that described above, except that DMP (28.2 g, $0.1 \mathrm{~mol})$ was added rather than SAM.

\subsection{Synthesis of poly (DMP-SAM-SA, PSiS) and poly (SAM-SA, SiS)}

Polymerization was conducted using a method similar to that described above.

\subsection{Methods and measurements}

The composition of the synthesized copolymers was determined using elementary analysis (EA) and ${ }^{1} \mathrm{H}-\mathrm{NMR}$ investigation. The ${ }^{1} \mathrm{H}-\mathrm{NMR}$ spectra of the monomer and copolymers were recorded using a Bruker AM400 NMR spectrometer, with $\mathrm{CDCl}_{3}$ as the solvent and tetramethylsilane as the internal standard. Finally, the molecular weight of the synthesized copolymer was determined by gel permeation chromatography (JASCO RI-1530, PU-1580).

The AW properties of the oils that contained synthesized copolymer were assessed by machine testing under the following conditions: rotation rate, $700 \mathrm{rpm}$; test duration, $120 \mathrm{~min}$; load, $200 \mathrm{~kg}$. The oil temperature, electrical contact resistance and frictional coefficient between the two metal surfaces were recorded. Following AW testing, the frictional pairs, shown in Fig. 1, were washed in pure acetone, and the weight loss was measured using the difference between the weights of the tested pieces before and after AW tests. Furthermore, a Brukfield viscosity meter was used to examine the viscosity of oils with added synthesized copolymer.

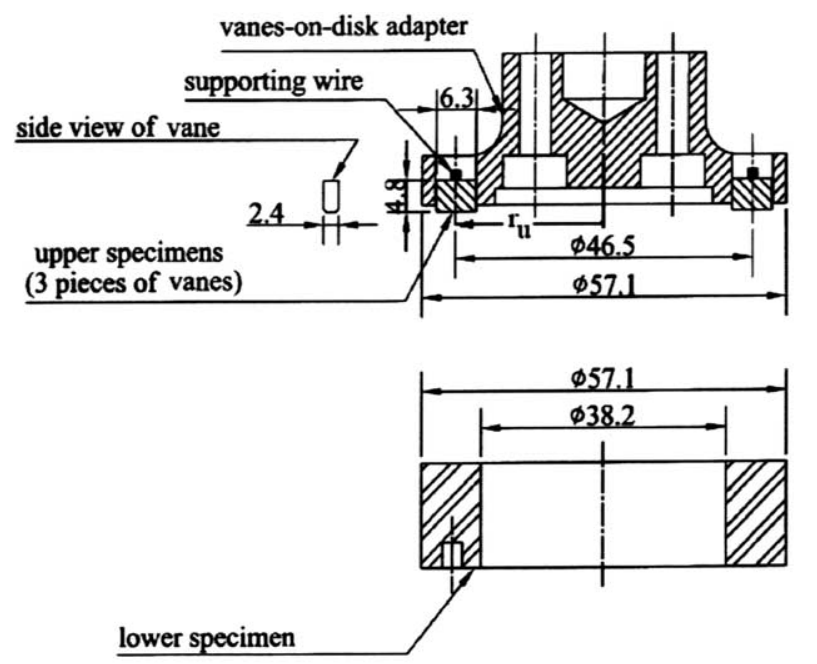

Unit: mm
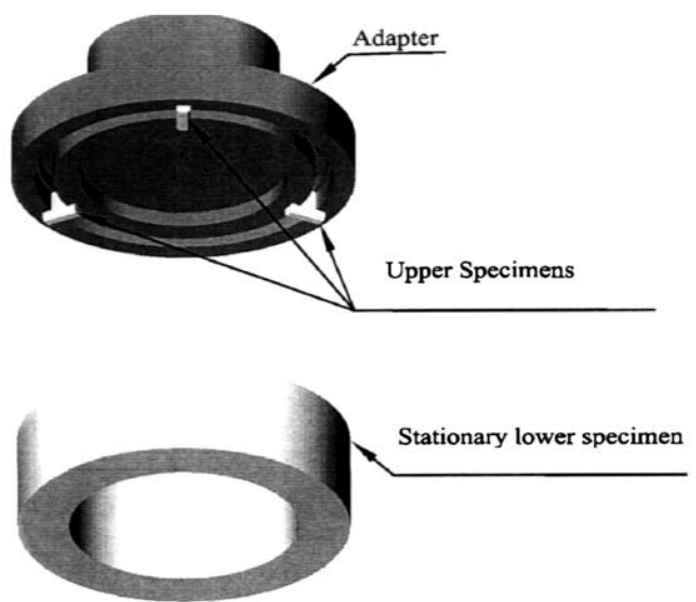

Fig. 1. The frictional pair used in testing machine for the AW behavior investigation, cited from the paper of Professors Chu and Lin [17]. 


\section{Results and discussion}

\subsection{Synthesis and characterization of desired copolymers}

This work synthesized and characterized several new copolymers, and then added them to base oil for further AW testing. SA was copolymerized with DMP, SAM, and DAEA monomers to prepare the polysiloxane-containing copolymers ( $\mathrm{SiS}$ ), polysiloxane-amino-containing copolymers (SiNS), thiophosphate-amino-containing copolymers (PNS), and thiophosphate-polysiloxane-containing copolymers (PSiS), respectively, as displayed in Scheme 1. Finally, the solubility of each synthesized copolymer in base oil was assessed.

For the SiNS copolymer, chemical shifts at $\delta=0.1 \mathrm{ppm}$ ( $\mathrm{Si}-\mathrm{CH}_{3}$ on polysiloxane), $0.85 \mathrm{ppm}$ (terminal $\mathrm{CH}_{3}$ on stearyl group), and $7.2 \mathrm{ppm}$ (phenyl group on polysiloxane) were observed in the ${ }^{1} \mathrm{H}-\mathrm{NMR}$ spectrum (Fig. 2). The figure illustrates that the polysiloxane and stearyl groups were included by the SiNS copolymer. Furthermore, the molar ratios of SAM and SA monomer in polysiloxane-containing copolymers can also be obtained from the ratio of the area under the peaks at $0.85 \mathrm{ppm}$ (terminal $\mathrm{CH}_{3}$ on SA) and $7.2 \mathrm{ppm}$ (phenyl group on polysiloxane) in the ${ }^{1} \mathrm{H}-\mathrm{NMR}$ spectrum. The molar ratios of DMP and DAEA monomer in these synthesized copolymers can be calculated because the amounts of sulfur in thiophosphate-containing copolymer and of nitrogen in amino-containing copolymer can be determined by EA.

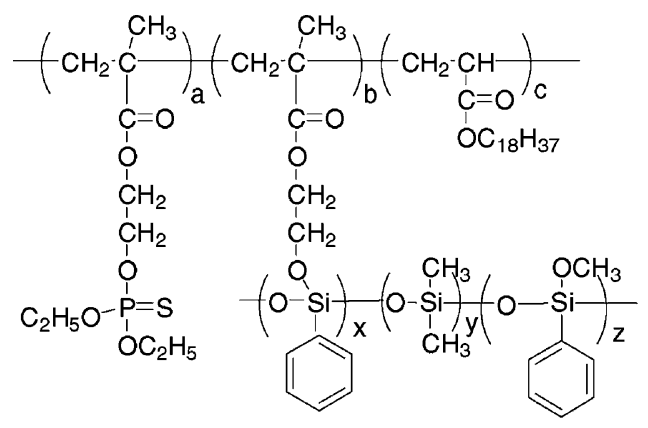

PSiS

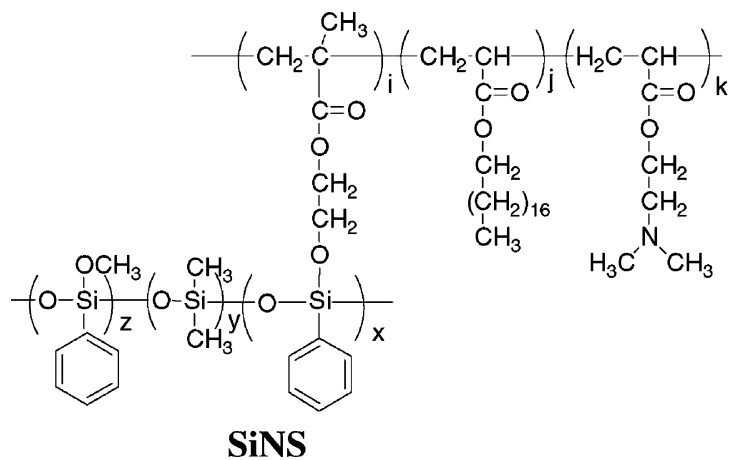

The molar ratios of the feeding monomers of SAM:SA, DMP:SAM:SA, SAM:DAEA:SA, and DMP:DAEA:SA in polymerization were adjusted several times, and only copolymers obtained with the desired monomer ratios were selected. The copolymers $\mathrm{SiS}$, PSiS, SiNS, and PNS with molar ratios of SAM:SA close to 1:3 and of DMP:SAM:SA, SAM:DAEA:SA, and DMP:DAEA:SA close to 1:1:3 were thus used for further investigation with a comparable base. Table 1 lists the molar ratios of distinct monomers and the number average molecular weight $(\overline{\mathrm{Mn}})$ of all the synthesized copolymers.

\subsection{Polymer behaviors on metal surfaces}

An AW machine was utilized to investigate AW properties by measuring the oil temperature, frictional coefficient, and electrical contact resistance between the two metal surfaces under a $200 \mathrm{~kg}$ load at $700 \mathrm{rpm}$ over $2 \mathrm{~h}$. The results were plotted against operating time, as shown in Figs. 3-6.

Fig. 3 plots the oil temperature versus operating time for the SiS, SiNS, PNS, and PSiS copolymers and the base oil. The oil temperature during testing was lower for the thiophosphate-containing copolymers PSiS and PNS than for the other synthesized copolymers. This finding was consistent with previous investigations by the current authors. A chemical adsorption layer may be generated for the oil with thiophosphate-containing copolymers, and the copolymers performed similarly on metal surfaces to EP additives in oil.
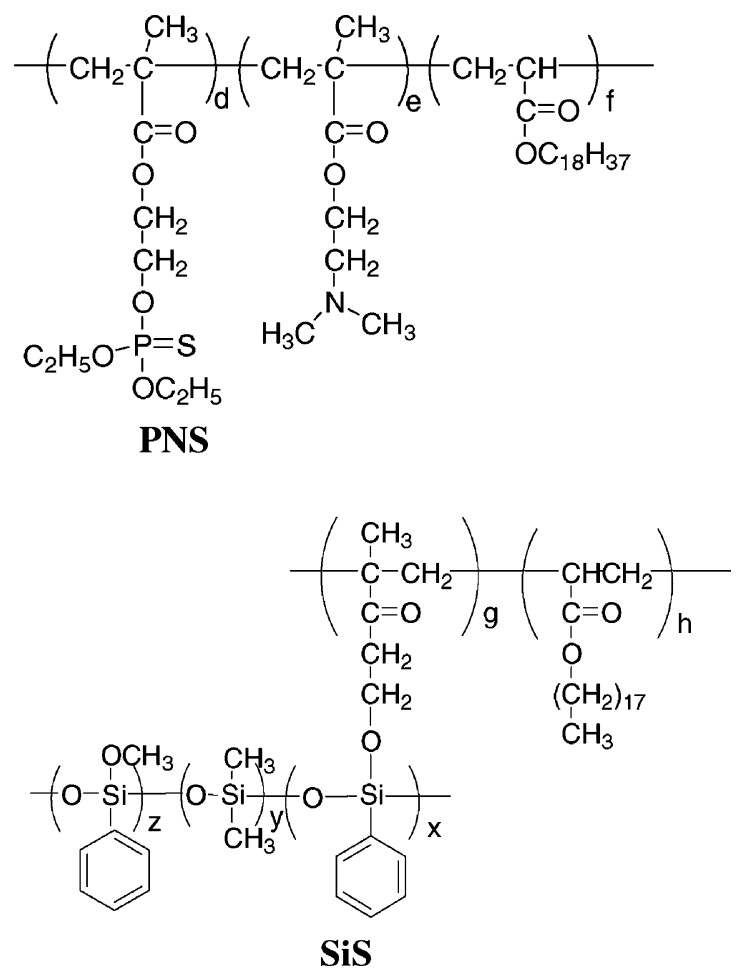

Scheme 1. The synthesized copolymers PSiS, PNS, SiNS, and SiS. 


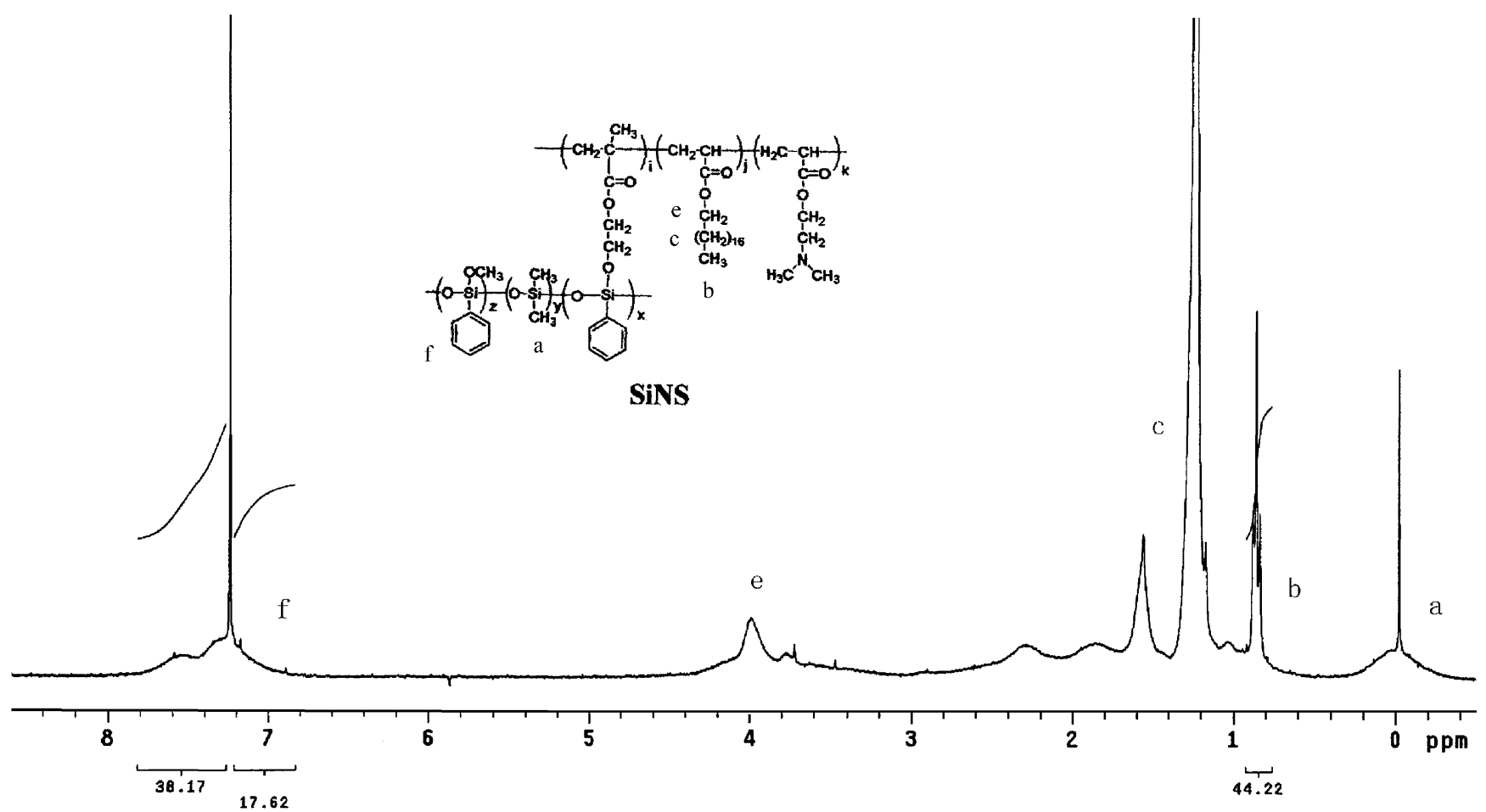

Fig. 2. ${ }^{1} \mathrm{H}-\mathrm{NMR}$ spectrum of SiNS copolymer.

The window of the operating period was expanded to $0-2000 \mathrm{~s}$ in Fig. 4 to highlight the variation in oil temperature at the beginning of the AW test. Fig. 5 plots the frictional coefficient between the two metal surfaces against operating time. In Figs. 4 and 5, the oil temperature with the SiNS copolymer was lowest for an operating period of $0-500 \mathrm{~s}$. The frictional coefficient of the SiNS copolymer also decreased smoothly from 0 to $500 \mathrm{~s}$, but increased thereafter. Therefore, SiNS adsorbed physically onto metal surfaces at the lower temperature but desorbed from those surfaces as the oil temperature increased. The oil temperature and frictional coefficient with the addition of SiNS, was significantly lower than with the addition of PNS copolymer that contained additional amino groups, before thiophosphate-containing copolymer PNS adsorbed onto the metal surfaces. This phenomenon suggests that the lower oil temperature and smaller frictional coefficient of the metal surfaces resulted from the presence of the polysiloxane group in the SiNS copolymer.

However, the frictional coefficient, with added SiNS copolymer, on metal surfaces exceeded that of PSiS, which is a thiophosphate-polysiloxane-containing copolymer. The oil temperature with SiNS remained lower than that of PSiS, because the physically adsorbed SiNS copolymer, which contained amino groups, continued to adhere to the metal surfaces during the initial stage of the AW test. Furthermore, copolymer PSiS adhered to metal surfaces, because of chemical adsorption [11]. This result demonstrated that these two polysiloxane-containing copolymers that settled

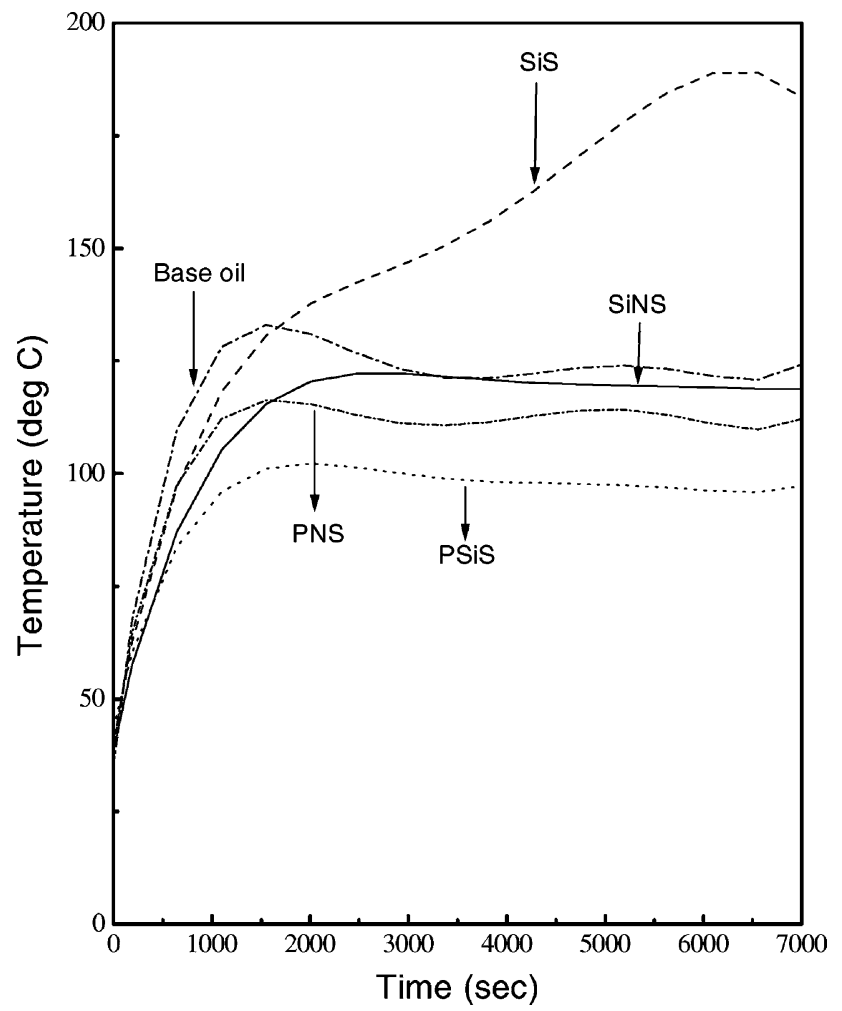

Fig. 3. Temperature of oil added with synthesized polymer and compared with base oil as a function of operating time $(0-7000 \mathrm{~s})$. 


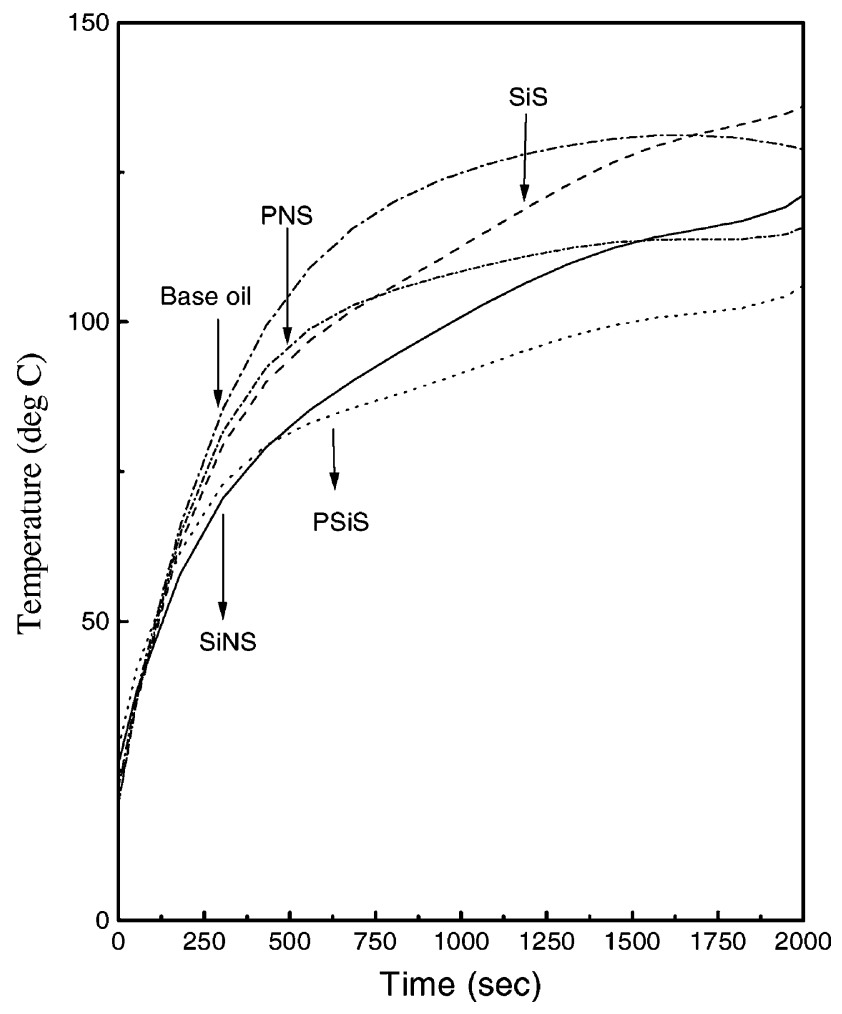

Fig. 4. Temperature of oil added with synthesized polymer and compared with base oil as a function of operating time (0-2000s).

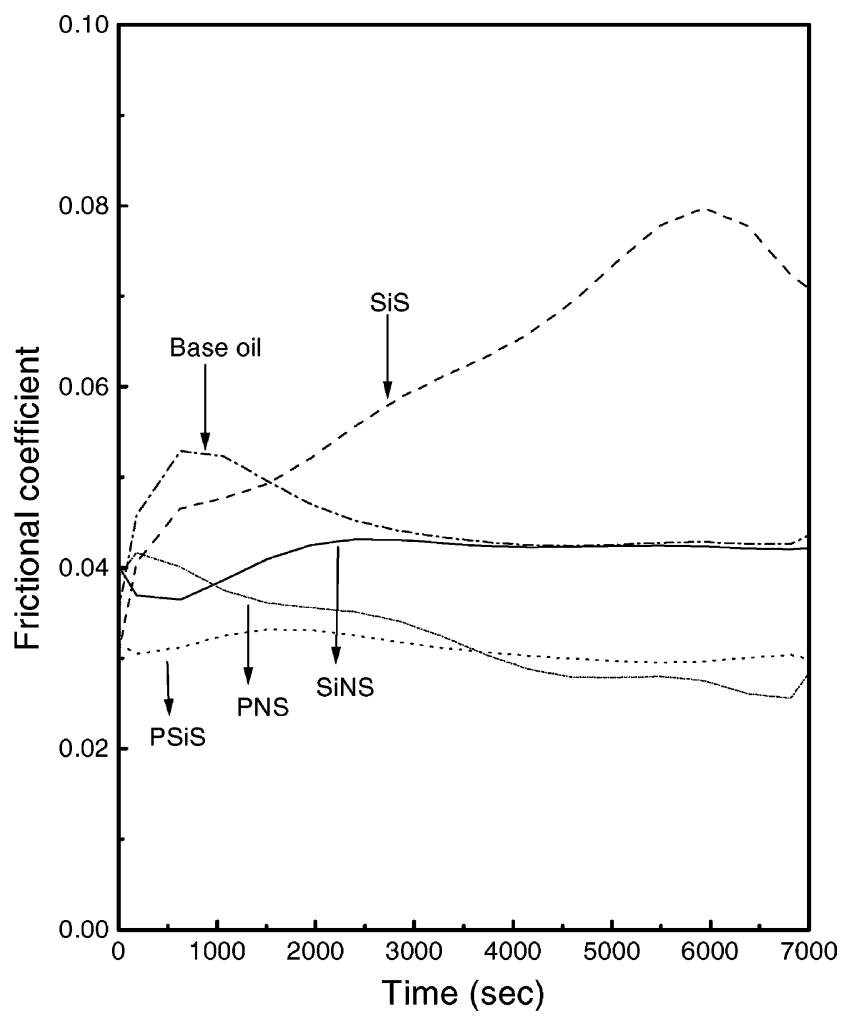

Fig. 5. Frictional coefficient between the two metal surfaces lubricated by the oil added with synthesized polymer and compared with base oil as a function of operating time. on the metal surfaces reduced the frictional coefficient and limited the increase in the oil temperature by the presence of the polysiloxane group.

An electrical contact resistance device was used to measure the electrical contact resistance between the two metal surfaces as a function of operating time and thus investigate the insulation of the adsorption layer on the surfaces. In Fig. 6, the contact resistance between the two metal surfaces of oil with added PNS copolymer increased from $500 \mathrm{~s}$, but declined thereafter, because of the chemical adsorption of the thiophosphate group onto metal surfaces. This phenomenon might also have improved the AW performance, as shown in Fig. 5, where the frictional coefficient of PNS declined after an operating time of $500 \mathrm{~s}$. However, the contact resistance of PSiS copolymer increased after an operating time of $2000 \mathrm{~s}$. This phenomenon indicates that a chemical adsorption layer was formed after $2000 \mathrm{~s}$ in a system of oil to which thiophosphate-containing copolymer was added. This formation of an adsorption layer controlled the increase in the oil temperature, and the frictional coefficient after 2000 s, as shown in Figs. 3 and 5. However, the oil temperature and frictional coefficient with the PSiS copolymer were both lower than those of other synthesized copolymers, before the chemical adsorption of the PSiS copolymer onto the metal surfaces at the start $(0-500 \mathrm{~s})$ of the AW investigation. Consequently, polysiloxane plays a major role in improving AW performance.

The weight loss of a frictional pair, according to the AW test, is generally decreased when an adsorption layer is formed on a metal surface. How the weights of the AW test pieces differed before and after testing was measured for the base oil and for oils with added copolymer SiS, PSiS, SiNS, or PNS. Table 1 lists the measurement results. The results clearly indicate that the weight loss values of thiophosphate-containing copolymers are significantly lower than those of the base oil, particularly in the case of copolymer PSiS.

\subsection{Viscosity study}

Higher viscosity is well known to enhance AW performance $[1,2]$. This work investigated the viscosities of the oils to which synthesized copolymer was added at 20,80, and $150^{\circ} \mathrm{C}$, and the measurement results are listed in Table 1 . The viscosity of the oils containing synthesized copolymer at $20^{\circ} \mathrm{C}$ exceeded that of base oil. As the oil temperature increased, the viscosities of the base oil and oils with synthesized copolymers decreased to ca. $20 \mathrm{cp}$ at $80^{\circ} \mathrm{C}$ and 5-6 cp at $150^{\circ} \mathrm{C}$. However, the viscosities of the oils with PSiS and SiNS were lower than those of oil containing other synthesized copolymers at $20^{\circ} \mathrm{C}$. These measurement results verify the above and demonstrate that oil temperature and frictional coefficient were not influenced by viscosity of oil, but significantly reduced by the presence of polysiloxane-containing copolymers. 

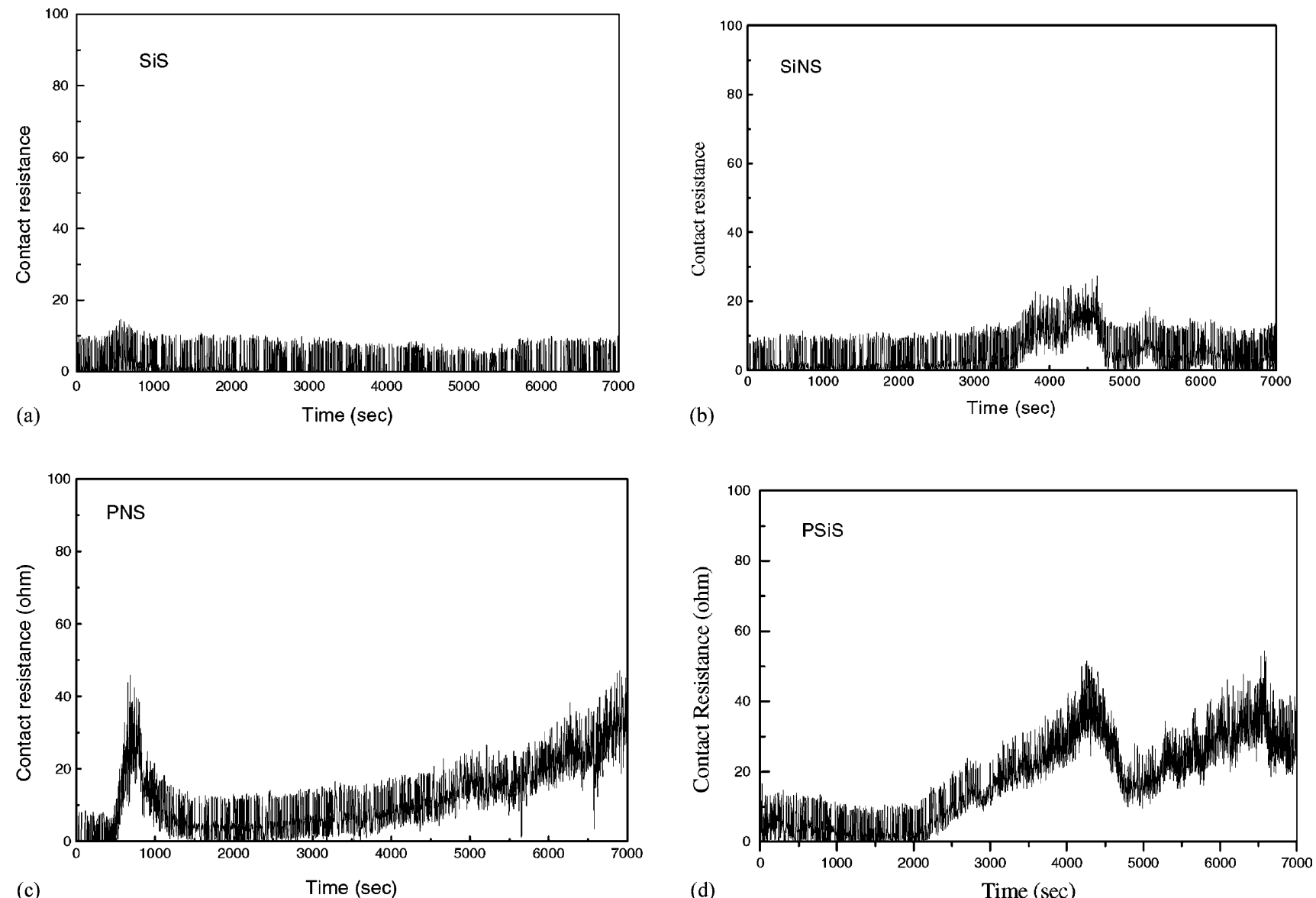

Fig. 6. Electrical contact resistance between the two metal surfaces lubricated by the oil added with synthesized polymer and compared with base oil as a function of operating time: (a) SiS; (b) SiNS; (c) PNS; (d) PSiS.

Table 1

The composition and average molecular weight for SiS, PSiS, SiNS and PNS copolymers, and the viscosity of oil and the wear amount of frictional pair after AW tests for oil added with these copolymers

\begin{tabular}{|c|c|c|c|c|c|c|c|}
\hline \multirow[t]{2}{*}{ Polymeric additive } & \multirow[t]{2}{*}{ Monomer units } & \multirow[t]{2}{*}{ Molar ratios in copolymer } & \multirow[t]{2}{*}{$\overline{\mathrm{Mn}}$} & \multirow[t]{2}{*}{ Wear amount ${ }^{\mathrm{a}}(\mathrm{mg})$} & \multicolumn{3}{|c|}{ Viscosity $^{\mathrm{b}}(\mathrm{cp})$} \\
\hline & & & & & $20^{\circ} \mathrm{C}$ & $80^{\circ} \mathrm{C}$ & $150^{\circ} \mathrm{C}$ \\
\hline $\mathrm{SiS}$ & SAM:SA & $1.09: 3$ & 2482 & 17.56 & 670 & 19 & 4.9 \\
\hline PSiS & DMP:SAM:SA & $0.84: 0.97: 3$ & 2673 & 0.22 & 382 & 19 & 5.9 \\
\hline SiNS & SAM:DAEA:SA & 0.99:0.95:3 & 2581 & 2.36 & 473 & 19 & 6.2 \\
\hline PNS & DMP:DAEA:SA & $1.10: 1.18: 3$ & 3451 & 0.71 & 880 & 21 & 6.2 \\
\hline
\end{tabular}

${ }^{a}$ The wear amount of metal for base oil was $4.53 \mathrm{mg}$.

${ }^{\mathrm{b}}$ Viscosity of base oil was 322,21 , and $5.2 \mathrm{cp}$ for 20,80 , and $150^{\circ} \mathrm{C}$, respectively.

\section{Conclusions}

The copolymers, SiS, SiNS, PNS, and PSiS, were synthesized by free radical copolymerization, and characterized by ${ }^{1} \mathrm{H}-\mathrm{NMR}$ and EA. The AW behaviors of the synthesized copolymers as an oil additive in base oil were assessed with a wear tester. Meanwhile, the performance of these synthesized copolymers at an oil/metal interface under a high load and rotary velocity was examined by measuring the oil temperature, frictional coefficient, and electrical contact resistance between the two metal surfaces. This work on AW performance found that the frictional coefficient between the two metal surfaces was reduced and the oil temperature was limited by chemical adsorption because of the presence of a thiophosphate-containing copolymer PNS or PSiS. Before chemical adsorption, both the oil temperature and the frictional coefficient of copolymer PSiS were lower than those of other synthesized copolymers. 
This was resulted from polysiloxane group provided by the thiophosphate-polysiloxane-containing copolymer. This phenomenon did not follow from the variation in viscosity testing because of the addition of copolymers, but from the polysiloxane group, which can enhance the AW characteristics. Furthermore, the copolymer SiNS adsorbed physically onto metal surfaces when the oil temperature was low and desorbed from metal surfaces as the temperature increased. The two polysiloxane-containing copolymers PSiS and SiNS that settled on the metal surfaces reduced the frictional coefficient and limited the increase in the oil temperature by the presence of the polysiloxane group.

\section{Acknowledgements}

The authors would like to thank Professor Jen-Fin Lin of the Department of Mechanical Engineering, National Cheng Kung University, for the technical assistance in determining AW properties, and the National Science Council for their general financial support of this research.

\section{References}

[1] T. Fujimoto, New Introduction to Surface Active Agents. Sanyo Chemical Industry, Japan, 1985, pp. 290-297.

[2] V. Štêpina, V. Veselý, Lubricants and Special Fluids. Elsevier, Czechoslovakia, 1992, pp. 381-393.

[3] H. Zinke, R. Schumacher, Tribol. Int. 30 (3) (1997) 199-208.

[4] H. Zinke, R. Schumacher, Wear 179 (1994) 45-48.

[5] T. Ohmori, K. Kitamura, A. Danno, M. Kawamura, Tribol. Trans. 34 (3) (1991) 458-463.

[6] D. Yang, Q. Xue, X. Zhang, X. Ding, W. Lin, Wear 193 (1996) 66-72.

[7] J.N. Cutler, J.H. Sanders, P.J. John, G. DeStasio, B. Gilbert, K. Tan, Wear 236 (1999) 165-178.

[8] T. Ren, G. Sha, L. Zhng, J. Xia, W. Liu, Q. Xue, J. Soc. Trib. Lub. Eng. 53 (1997) 39.

[9] R. Sarin, D.K. Tuli, V. Martin, M.M. Rai, A.K. Bhatnagar, J. Soc. Trib. Lub. Eng. 53 (1997) 21-26.

[10] J. Zhang, W. Liu, Q. Xue, Wear 231 (1999) 279-284.

[11] S.C. Ni, P.L. Kuo, Submitted for publication.

[12] G. Caporiccio, P.M. Cann, H.A. Spikes, Wear 193 (1996) 261-268.

[13] M.K. Mishra, R.G. Saxton, Chemtech (1995) 35-41.

[14] W. Liu, L. Hu, Z. Zhang, Thin Solid Films 127 (1995) 88-91.

[15] G.C. Smith, J.C. Bell, Appl. Surf. Sci. 144 (1999) 222-227.

[16] S.C. Ni, P.L. Kuo, Submitted for publication.

[17] H.Y. Chu, J.F. Lin, Wear 239 (2000) 126. 